Vietnam Journal of Mechanics, NCST of Vietnam Vol. 22, 2000, No 2 (101 - 110)

\title{
SHOCK ADIABAT ANALYSIS FOR THE MIXTURE OF LIQUID AND GAS OF TWO COMPONENTS
}

\author{
DUONG NGOC HAI \\ Institute of Mechanics, NCST of Vietnam \\ NGUYEN VAN TUAN \\ Technical Industrial University of Thai Nguyen
}

\begin{abstract}
The liquid and gas mixture is met in many natural and industrial processes. In the paper, the investigation results of shock waves, propagated in the liquid and two-component gas (one component is neutral, the other is condensed) mixture, are presented. The influence of the relative gas ratios, initial gas volume, wave intensity etc. on the main wave characteristics, is analyzed and illustrated as examples for two mixtures. The first is a mixture of water and gas (air and water vapour). The second is a mixture of crude oil and gas (one component is soluble, the other is insoluble).
\end{abstract}

\section{Introduction}

A heterogeneous medium is supposed to be a two-phase one: a liquid phase and a gas phase, in which the gas phase consists of two components, one of which is neutral (non-condensable, insoluble) gas and the other is vapour (or soluble gas). This medium can be seen in the following fields:

- In the energy industry, this medium is used as a heat carrying medium.

- In the oil exploitation, transportation and processing industry, gas appears when there are changes of pressure and temperature, because crude oil is normally a mixture of various components in which $\mathrm{CH}_{4}, \mathrm{C}_{2} \mathrm{H}_{6}, \ldots$ evaporate easily at a normal temperature and pressure.

Besides this kind of a mixture can also be seen in chemical engineering and natural processes [1-3].

Shock waves in liquid with bubbles of an insoluble and non-condensable gas have been investigated theoretically and experimentally by Nigmatulin, Khabeev and Shagapov (1974); Nigmatulin and Shagapov (1974); Aidagulov, Khabeev and Shagapov (1977). Shock waves in liquid with bubbles of easily soluble gas have been investigated theoretically and experimentally by Terushige Fujii and Koji Akagawa (1991); V. E. Donstrov and B. G. Pokusaev (1998).

The propagation of waves in a liquid with vapour bubbles has been investigated by Nigmatulin, Shagapov, Vakhitova and Shikhmuzaeva (1982); Duong Ngoc Hai, Nigmatulin and Khabeev (1982, 1984, 1988); Duong Ngoc Hai (1987); 
S. Morioka (1989); Y. Kobayashi (1989).

Let us consider a mixture of liquid and gas bubbles of two components (air and water vapour) which, for example, is held in a pipe. Let a stationary shock wave move towards a place where there is a closed pipe valve or the bottom of the pipe. The closed pipe valve or the pipe bottom is proposed to be made by a perfectly solid material. This shock wave propagates in the mixture, acts on the valve or on the pipe bottom, reflects from it and moves backwards.

Although the mass of a gas phase is normally many times less than the mass of the mixture, its existence in the mixture can completely change motional and physical features of the medium. That is, the influence of the gas phase of the mixture on the velocity of the incident wave, on the intensity of the shock wave in the mixture, after being reflected from the solid wall (the close pipe valve or the bottom of the pipe) and etc. will be considered and reported in this paper.

\section{Symbols and basic equations}

In order to investigate the problems presented above, the following symbols are used:

$D$ - Velocity of wave;

$v$ - Velocity of mixture;

$\rho$ - Density of material;

$p$ - Pressure;

$x_{i}$ - Fraction of mass of $i^{\text {th }}$ phase;

$\alpha_{i}$ - Fraction of volume of $i^{\text {th }}$ phase; $0 \leq \alpha_{i} \leq 1$.

Also, the following indexes are used:

- Subscripts:

1 - Referring to liquid phase; 2 - Referring to gas phase;

$v$-Vapour; $g$ - Gäs;

0 - Values of variable at initial state.

- Superscripts:

(1) - Equilibrium state of mixture behind the incident wave.

(2) - Equilibrium state of mixture behind the reflected wave.

0 - True density.

At the same time, it is assumed that in front of the incident wave and after reflection of the wave from the valve of the pipe or the pipe bottom the medium is at rest $\left(v_{0}=v^{(2)}=0\right)$; behind the incident wave it acquires velocity $v^{(1)}$.

There is no change in temperature. Because of the release of heat which is mainly caused by vapour condensation in the bubbles, and of the transformation of the kinetic energy of small-scale motion around the bubbles into the heat, the temperature in the mixture, indeed, increases. However, even for the case, when the volume fraction of the gas phase is many times more than the volume fraction of the liquid phase and the kinetic energy of small-scale motion is completely transformed into the heat, the increase of the temperature of mixture is very small (Duong Ngoc Hai et al. 1988). 
For example, under normal conditions, $p_{0}=0.1 \mathrm{MPa}$, the volume fraction of gas phase $\alpha_{20}$ equals 0.8 (gas consists of $70 \%$ of vapour and $30 \%$ of air), the increase of temperature of the mixture of water and air-water vapour bubbles is low:

$$
\Delta T \sim \frac{\alpha_{v 0} \rho_{v}^{0} \ell}{\alpha_{10} \rho_{1}^{0} C_{\ell}} \sim 1^{\circ} K,
$$

here $\ell$ is the specific heat of vaporization, $C_{\ell}$ is the thermal capacity of water.

For the medium under consideration, the laws of conservation of masses and momentum of the mixture, in the case of an incident wave with velocity $D^{(1)}$ and a reflected wave with velocity $D^{(2)}$ (relative to the valve or the bottom of the pipe), have the following forms

$$
\begin{aligned}
& \rho_{0} D^{(1)}=\rho^{(1)}\left(D^{(1)}-v^{(1)}\right), \\
& p^{(1)}-p_{0}=\rho_{0} D^{(1)} v^{(1)}, \\
& \rho^{(1)}\left(D^{(2)}+v^{(1)}\right)=\rho^{(2)} D^{(2)}, \\
& p^{(2)}-p^{(1)}=\rho^{(1)}\left(D^{(2)}+v^{(1)}\right) v^{(1)} .
\end{aligned}
$$

Because $\alpha_{i}$ is the volume fraction of $i^{\text {th }}$ phase, we have:

$$
\dot{\rho}^{(i)}=\alpha_{1}^{(i)} \rho_{1}^{O(i)}+\alpha_{2}^{(i)} \rho_{2}^{0(i)} .
$$

From (2.5) we have:

$$
\rho^{(1)}=\alpha_{1}^{(1)} \rho_{1}^{O(1)}+\alpha_{2}^{(1)} \rho_{2}^{O(1)} \Leftrightarrow \rho^{(1)}=\rho_{1}^{0(1)}+\alpha_{2}^{(1)}\left(\rho_{2 g}^{O(1)}+\rho_{2 v}^{O(1)}-\rho_{1}^{O(1)}\right) .
$$

Because $x_{i}$ is the mass fraction of $i^{\text {th }}$ phase, we also have the following expression:

$$
x_{i}=\frac{\alpha_{i} \rho_{i}^{0}}{\rho_{0}} \Rightarrow x_{g}=\frac{\alpha_{20} \rho_{g 0}^{0}}{\rho_{0}}=\frac{\alpha_{2}^{(1)} \rho_{g}^{o(1)}}{\rho^{(1)}}=\frac{\alpha_{2}^{(2)} \rho_{g}^{o(2)}}{\rho^{(2)}}=\text { const. }
$$

From (2.7) we have:

$$
x_{g}=\frac{\alpha_{2}^{1} \rho_{g}^{O(1)}}{\alpha_{1}^{(1)} \rho_{1}^{0(1)}+\alpha_{2}^{(1)}\left(\rho_{g}^{0(1)}+\rho_{v}^{0(1)}\right)} .
$$

So it follows:

$$
\alpha_{2}^{(1)}=\frac{\rho_{1}^{0(1)}}{\rho_{1}^{0(1)}-\rho_{v}^{0(1)}+\frac{1-x_{g}}{x_{g}} \rho_{g}^{0(1)}} .
$$




\section{Results and discussion}

Based on the equations presented above $(2.1),(2.2)$, we can obtain the expression for the incident wave velocity:

$$
D^{(1)^{2}}=\frac{p^{(1)}-p_{0}}{\rho_{0}} \frac{1}{1-\frac{\rho_{0}}{\rho_{1}^{O(1)}}\left[\frac{\alpha_{2 g}^{(1)}}{\rho^{(1)}}\left(\rho_{1}^{O(1)}-\rho_{g}^{O(1)}-\rho_{v}^{O(1)}\right)+1\right]}
$$

Therefore, the velocity of wave propagation $D^{(1)}$ in a mixture depends not only on physical properties of phases, but also on the phase ratio. Through this expression, we can easily find that when the phase ratio changes, although this change may be small and the intensity of shock waves not very high, there will be a remarkable change in the features of motion waves.

Applying the above results, to investigate a liquid-gas mixture in a special case: supposing that the liquid is compressible, the gas phase consists of only vapour, and the volume fraction of the gas phase is smaller than volume fraction of the liquid phase, i.e.

$$
\rho_{g 0}^{0}=0, \quad \alpha_{2 g}^{0}=\alpha_{2 g}^{(1)}=0, \quad \alpha_{2}<\alpha_{1} .
$$

From the expression (3.1) with the conditions listed above, it follows

$$
D^{(1)^{2}}=\frac{\Delta p^{(1)}}{\alpha_{10} \rho_{10}^{0}} \cdot \frac{\rho_{10}^{0}+\frac{\Delta p^{(1)}}{\rho_{10}^{0} a_{\ell}^{2}}}{1+\frac{\Delta p^{(1)}}{\alpha_{20} \rho_{10}^{0} a_{\ell}^{2}}}
$$

where: $\Delta p^{(1)}=p^{(1)}-p_{0}, a_{\ell}$ - velocity of sound propagation in liquid.

The expression (3.2) coincides with the result calculated in [3].

The calculation results allow to analyse the influences of factors, such as the relative density of the gas component, the intensity of the incident wave and the initial volume of gas phase, on the main features of shock wave.

In Fig. 1 and also Fig. 2a-4a presented below, solid curves express processes happening to the mixture of water and gas of two components (air and water vapour), dash-doted curves express processes happening to the mixture of crude oil and gas of two components (one component is soluble, the other-air is insoluble).

According to the expression (3.1), the calculation results are described in Fig. 1. Curves in Fig. 1 show the dependence of the velocity of the incident wave $D^{(1)}$ on volume fraction $\alpha_{20}$ of gas phase, in which the gas phase consists of two components, one component is air gas (non-condensable, insoluble) and the other is vapour (or soluble gas). The incident wave velocity will be decreased quickly, 
while the volume fraction $\alpha_{20}$ of the gas phase is increasing. Curve 2' corresponds to the case of a mass fraction of soluble gas equal to $50 \%$ of the gas phase. Curves 1-3 correspond to the cases of mass fraction of vapour equal to $0 ; 50 ; 100 \%$ of gas phase, respectively. In Fig. 1 we have seen that the existence of soluble gas or vapour in the gas phase, doesn't have a great influence on the incident wave velocity in the mixture.

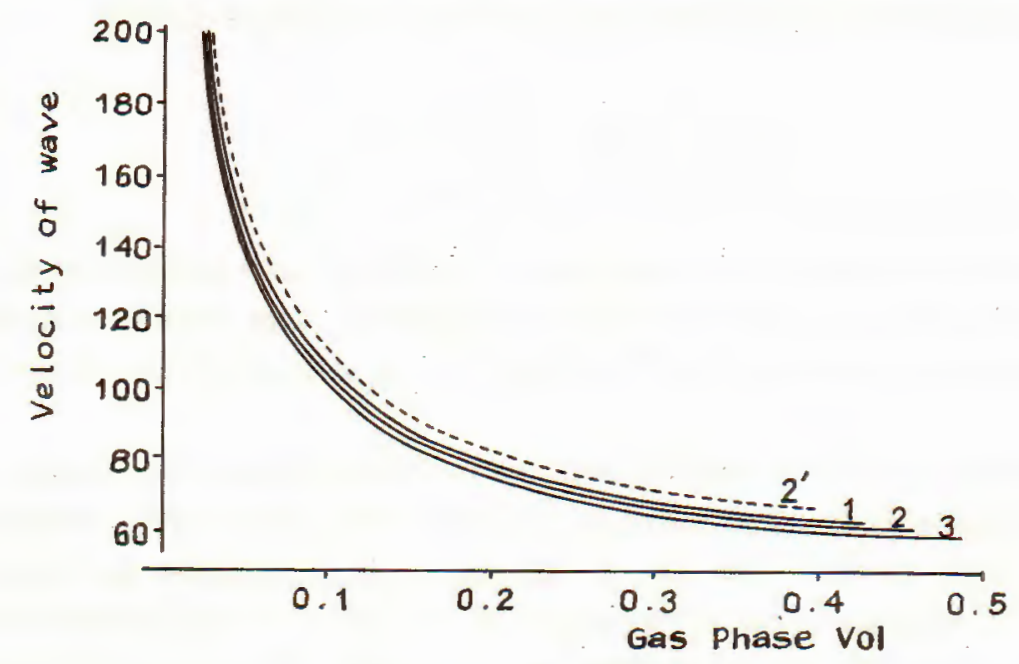

Fig. 1

First, the shock wave affects on the mixture and its velocity are determined by the above presented expression. After that, this wave acts on the bottom of the pipe and reflects from it and moves backwards. Based on equations (2.3), (2.4) and the above results (3.1), the parameters of the reflected wave can be expressed through parameters behind the incident waves.

$$
\frac{p^{(2)}}{p^{(1)}}=1+\frac{\rho^{(2)}}{\rho_{0}} \cdot \frac{\left(\rho^{(1)}-\rho_{0}\right)}{\left(\rho^{(2)}-\rho^{(1)}\right)} \cdot \frac{\left(p^{(1)}-p_{0}\right)}{p^{(1)}},
$$

here

$$
\begin{aligned}
& \rho^{(2)}=\alpha_{1}^{(2)} \rho_{1}^{O(2)}+\alpha_{2}^{(2)} \rho_{2}^{O(2)}, \\
& \alpha_{2}^{(2)}=\frac{\rho_{1}^{0(2)}}{\rho_{1}^{O(2)}-\rho_{v}^{O(2)}+\frac{1-x_{g}}{x_{g}} \rho_{g}^{O(2)}} .
\end{aligned}
$$

The formulae (3.3) permits to determine the pressure behind reflected wave $p^{(2)}$ when the values $p_{0}, p^{(1)}, p_{v 0}, \alpha_{20}$ are known and it can be rewritten as follows:

$$
F\left(p^{(2)}\right)=p^{(2)}-p^{(1)} \frac{\left(\rho^{(1)}-\rho_{0}\right)\left(\rho^{(1)}-\rho_{0}\right)}{\rho_{0}\left(1-\frac{\rho^{(1)}}{\rho^{(2)}}\right)}=0 .
$$


Combined with the expression of $\rho^{(1)}$ it follows:

$$
\frac{\rho_{0}}{\rho^{(i)}}=\frac{1}{1-\frac{\alpha_{20} \rho_{v}^{O(i)}\left(p_{0}-p_{v 0}\right)}{\rho_{0}\left(p^{(i)}-p_{v 0}\right)}}\left[\alpha_{20} \frac{p_{0}-p_{v 0}}{p^{(i)}-p_{v 0}}\left(1-\frac{\rho_{v}^{O(i)}}{\rho_{1}^{O(i)}}\right)+\frac{\rho_{0}-\alpha_{20} \rho_{g 0}^{0}}{\rho_{1}^{0(i)}}\right] .
$$

To solve the equation (3.4), Newton iteration method is used:

$$
p_{n+1}^{(2)}=p_{n}^{(2)}-\frac{F\left(p_{n}^{(2)}\right)}{F^{\prime}\left(p_{n}^{(2)}\right)} .
$$

Because it can easily be seen that function $F$ in (3.4) has continuous derivatives of first and second orders, at the same time they satisfy the Fourier condition, i.e. there is $p_{0}^{(2)}$ in $(0, \infty)$ satisfying $F\left(p_{0}^{(2)}\right) F^{\prime \prime}\left(p_{0}^{(2)}\right)>0$, where $p_{0}^{(2)}$ is a first iteration value $[5]$.

Solving equation (3.4) we can determine an enhancement of shock wave intensity in the liquid and two-component gas mixture, after being reflected from the closed pipe valve or the bottom of the pipe. In Fig.2a-4a graphs describe the dependence of pressure induced by shock wave, after being reflected by a perfectly solid wall (the valve or the bottom of the pipe). This pressure depends on dimensionless variables: the volume fraction $\alpha_{20}$ of gas phase, the dimensionless intensity of incident wave $P^{(1)}=p^{(1)} / p_{0}$, the relative compressibility of the medium compressibility of front and behind the incident wave $a u=p_{0} /\left(\phi_{10}^{0} \cdot a_{\ell}^{2}\right)$ and the mass ratio of soluble gas in the gas phase.

In Fig. $2 \mathrm{a}$, the diagram expresses a change of $p^{(2)} / p^{(1)}$ depending on the volume fraction $\alpha_{20}$ of the gas phase (including gas bubble and water vapour or soluble gas and insoluble gas). Curves 1-5 correspond to the cases of mass fraction of vapour equal to 100 (pure vapour); $80 ; 55 ; 35$ and $0 \%$ (pure (neutral) gas) of gas phase, respectively, at conditions $p_{0}=0.1 \mathrm{MPa}, p^{(1)}=1 \mathrm{MPa}$, i.e. $P^{(1)}=10$. Curve 1 and 5 coincide with the results calculated in [8]. In Fig. 2b curves $1-3$ express the change of $p^{(2)} / p^{(1)}$ depending on the volume fraction $\alpha_{20}$ of the gas phase (in which the gas phase consist of $55 \%$ of mass vapour and $45 \%$ of air) and the intensity shock wave $P^{(1)}$ while $P^{(1)}$ equals $20,10,7$.

In Fig. 3a, curves 1-5 express the change of $p^{(2)} / p^{(1)}$ in dependence on the intensity of incident wave $P^{(1)}$ in the case of the volume fraction of gas phase $\alpha_{20}$ equal $0.05 ; 0.025 ; 0.01 ; 0.005$ and 0.001 , respectively, at condition initial pressure $p_{0}=0.1 \mathrm{MPa}$. In this case the gas phase consists of $60 \%$ of vapour (or soluble gas) and $40 \%$ of neutral (non-condensable, insoluble) gas. In Fig. $3 \mathrm{~b}$, the graph expresses the change of $p^{(2)} / p^{(1)}$ in dependence on the intensity of incident wave $P^{(1)}$ in the case of volume fraction of gas phase $\alpha_{20}$ equals 0.01 , at condition initial pressure $p_{0}=0.1 \mathrm{MPa}$. Curves $1-5$ correspondent to the mass fraction vapour equal 100 (pure vapour); 75;50;30;0\% (pure air) of gas phase, respectively. 


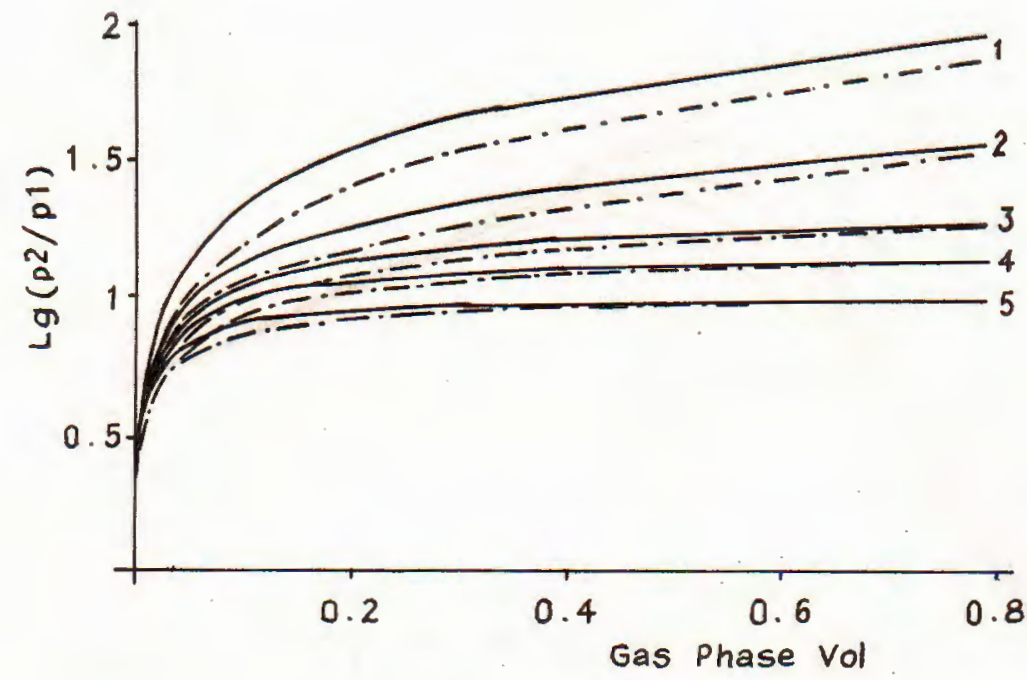

Fig. 2a

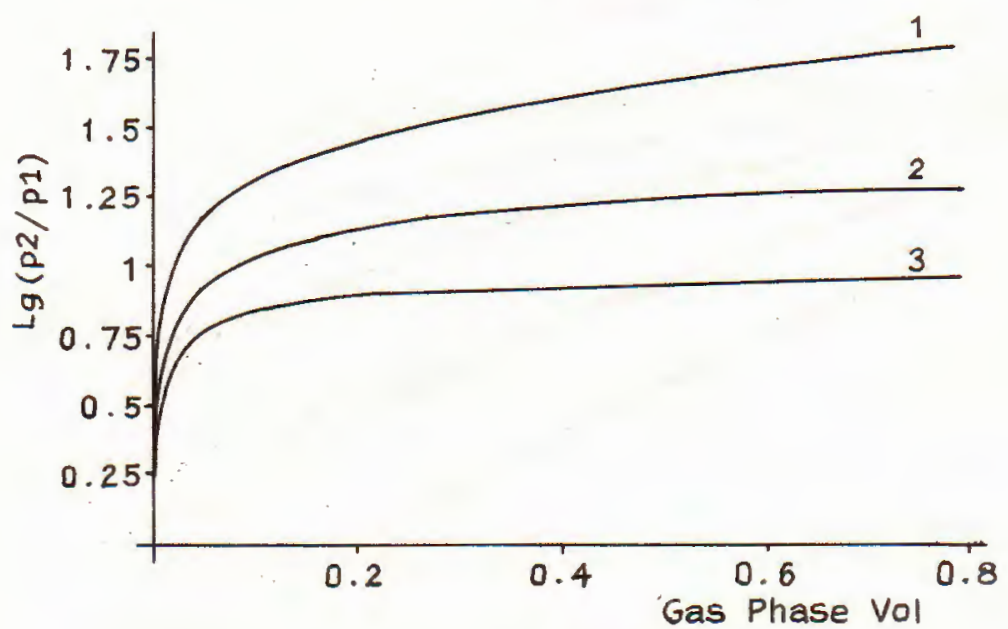

Fig. 2b

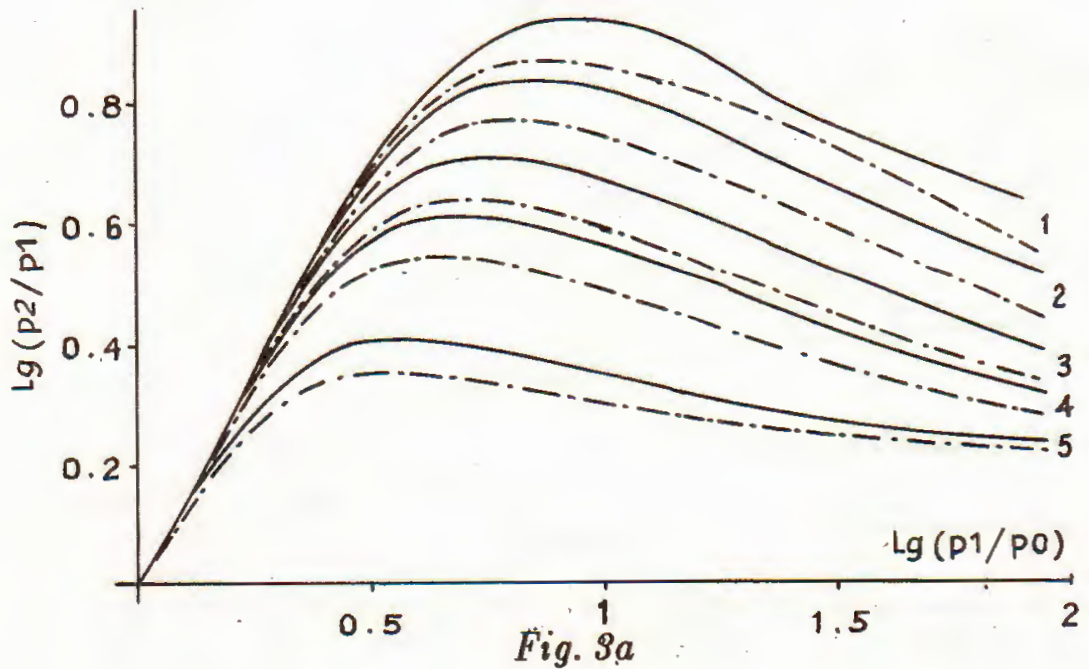




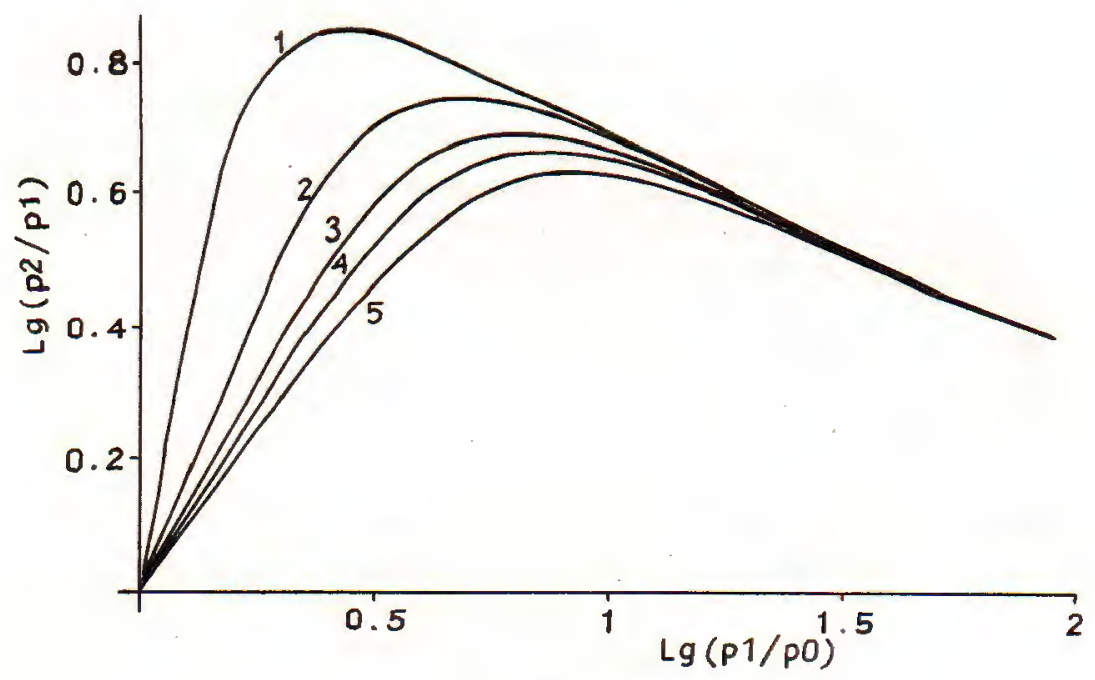

Fig. $3 b$
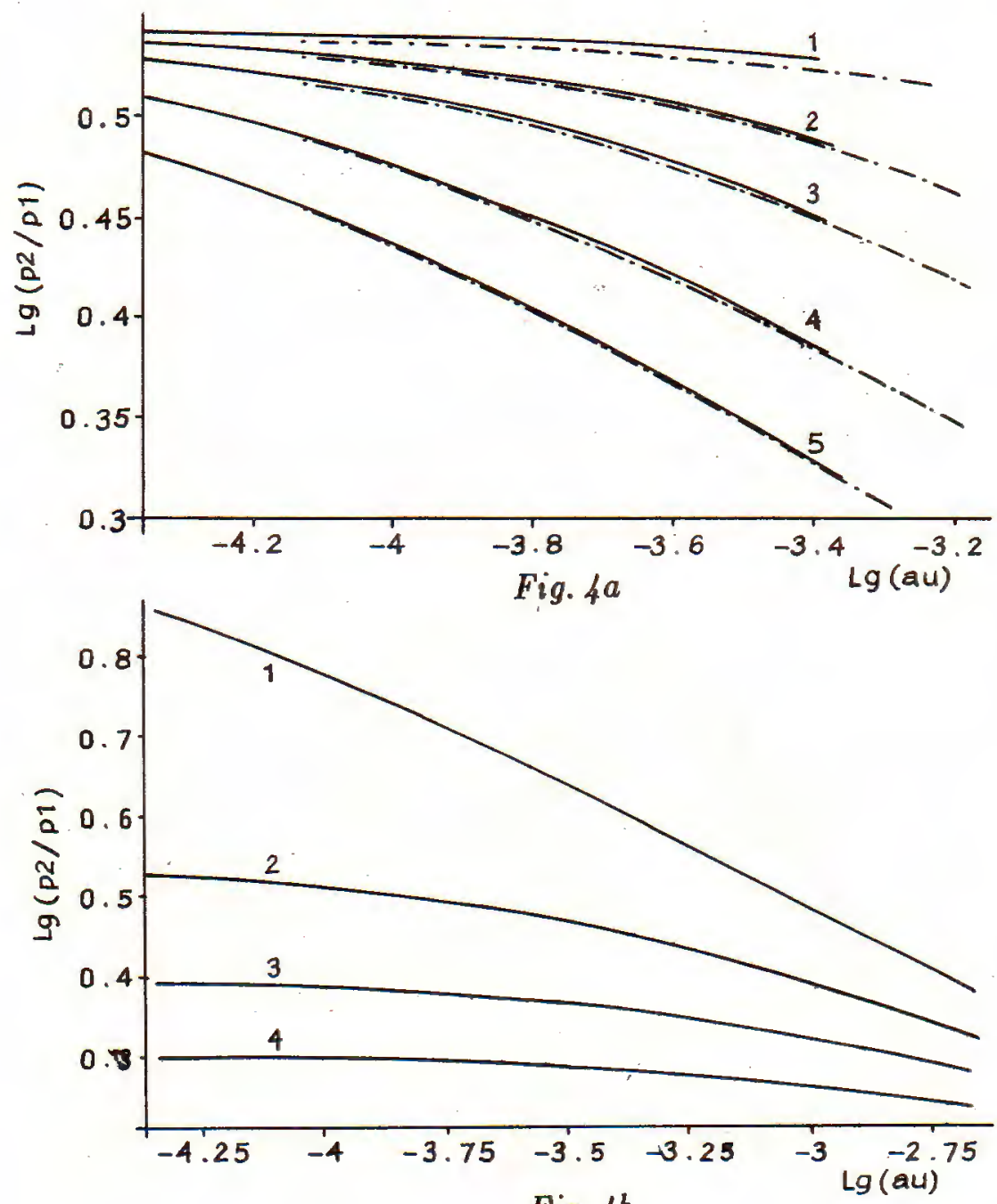

Fig. $4 b$ 
In Fig. 4a, curves 1-5 express the change of $p^{(2)} / p^{(1)}$ depending on dimensionless variable $a u=p_{0} /\left(\rho_{10}^{0} \cdot a_{\ell}^{2}\right)$ (through the change of initial (pressure) condition $\left.p_{0}\right)$ in the case of volume fraction of gas phase $\alpha_{20}$ equals $0.2 ; 0.05 ; 0.025 ; 0.01$ and 0.005 , respectively. In this case $P^{(1)}=p^{(1)} / p_{0}=$ const $=2$ and the gas phase consists of $75 \%$ of vapour (or soluble gas) and $25 \%$ of neutral (non-condensable, insoluble) gas. In Fig. $4 \mathrm{~b}$, graphs express the change of $p^{(2)} / p^{(1)}$ depending on dimensionless variable $a u$ in the case of volume fraction of gas phase $\alpha_{20}$ equal 0.025 . Curves $1-4$ correspondent to the mass fraction vapour equal to 100 (pure vapour) and 75, 50 and $0 \%$ (pure air) of the gas phase, respectively.

\section{Conclusions}

Heterogeneous media, in the form of liquid and two-components gas mixture, can be found in the energy industry, chemical engineering, natural processes, and especially in the oil exploitation, transportation and processing industry.

Through the above results, it can be found that the velocity of the incident wave will decrease rapidly, while the volume of the gas phase increases. The existence of the vapour or soluble gas in the gas phase doesn't have a great influence on the incident wave velocity. The incident wave velocity in the mixture of water and bubbles of air and vapour decreases more quickly than the incident wave velocity in the mixture of crude oil and bubbles of soluble and insoluble gas.

The structure and the properties of the medium influence, not only on the velocity of the incident wave, but also on the intensity of the reflection shock wave. This intensity of reflection shock wave will increase rapidly while the volume fraction of gas phase increases in the mixture. At the same time, the intensity of the shock wave depends on the ratio of mass fraction of the vapour (or soluble gas) or the mass fraction of air (or insoluble gas) to the gas phase. The enhancement of intensity of the shock wave in the case of the mixture of water and vapour is many times more than that of water and air. In identical condition of the pressure in the medium and the ratio of the volume fraction of the gas phase to the volume mixture, the intensity of the reflection shock wave in the cases of mixtures of liquid and two-component gas is of value between values of two cases the water-vapour and water-air mixture. Also the intensity reflection wave will increases while the mass fraction vapour increase in the gas phase.

The calculation results obtained, have pointed out that the condensation of the vapour (or soluble gas) in the gas phase which changes the structure of the mixture and its compressibility leads to an increase of the intensity of a pressure wave in the medium. This increase depends on the existence of mass vapour or soluble gas in the gas phase. The bigger the mass vapour of the mixture is, the bigger the increase of pressure in the mixture is. Also, the intensity of the incident wave has strongly affects the intensity of the shock wave, after being reflected from the solid wall.

Besides, the intensity of the shock wave depends on the relative compressibility of the mixture in front of and behind the incident wave. The existence of the 
vapour in the gas phase of the mixture, has a great influence on the compressibility of the mixture. The bigger the compressibility of the mixture is, the bigger the intensity of the shock wave becomes.

Acknowledgements. The research is completed with partial financial support from Vietnam National Basic Research Foundation in Natural Sciences, to which the authors would like to express their sincere thanks.

\section{REFERENCES}

1. Nigmatulin R. I. Dynamics of Multiphase System. Hemisphere, 1991.

2. Fujii T. et al. Water hammer phenomena in one-component two-phase bubbly flow. Proc. of the Int. Conf. on Multiphase Flow' 91-Tsukuba, 1991.

3. Nigmatulin R. I. Khabeev N. S. and Duong Ngoc Hai. Waves in liquid with vapour bubbles. J. Fluid Mech., Vol. 186, 1988.

4. Dontsov V. E., Pokusaev B. G. Experimental study of gas solution behind a shock wave in liquid with bubbles of easily solubble gas. Proc. of Int. Conf. on Multiphase Flow ICMF' 98, Lyon, France 1998.

5. Phan Van Hap, Nguyen Quy Hy, Hoang Duc Nguyen, Nguyen Cong Thuy. Calculation method. Hanoi, Vietnam, 1969.

6. Kobayashi Y. Vapour condensation behind a shock wave propagating through vapour liquid two-phase media. In: Adiabatic waves in liquid vapour system, IUTAM Symposium, Germany, 1989.

7. Morioka S. Wave propagation in flowing bubbly liquid. In: Adiabatic waves in liquid vapour systems, IUTAM. Symposium, Germany, 1989.

8. Duong Ngoc Hai. Shock wave in liquid with bubbles of gas or vapour, J. Mechanics No 4, 1987.

9. Aidagulov R. R., Khabeev N. S. and Shagapov V. Sh. Structure of the shock wave in a liquid with gas bubbles with allowance for unsteady interphase heat transfer. Zh. Prikl. Tekh. 1977.

Received February 15, 2000

PHÂN TÍCH ĐƯỜNG ĐOAN NHIÊT SÓNG XUNG KÍCH

TRONG HỖN HỢP CHẤT LỎNG VÀ KHÍ 2 THÀNH PHẦN

Hỗn hợp lóng-khí thường gặp trong nhiều quá trình tự nhiên và trong công nghiệp. Trong bài báo trình bày kết quả phân tích đoạn nhiệt sóng xung kích trong hổn hợp chất lóng và khí 2 thành phần: một thành phần trung tính, thành phần còn lại có thể ngưng tụ hay hòa tan. Đã phân tích sự ảnh hướng của tỷ lệ khí, thể tích khí ban đầu, cường độ sóng v.v... lên những đặc trưng chính của quá trình. Đã xem xét 2 hỗn hợp: nước-khí (khí gồm 2 thành phần: không khí và hơi nước) và dầu thô-khí (khí gồm 2 thành phần: khí hòa tan và khí trung tính).

Institute of Mechanics

264 Doi Can Str., Hanoi, Vietnam 